\title{
Holö, Andrej, Lederer, Klaus, Naumann, Matthias, Linke Metropolenpolitik. Erfahrungen und Perspektiven am Beispiel Berlin
}

\section{Antoine Laporte}

\section{(2) OpenEdition}

1 Journals

Édition électronique

URL : http://journals.openedition.org/ifha/6534

DOI : $10.4000 /$ ifha. 6534

ISSN : 2198-8943

Éditeur

IFRA - Institut franco-allemand (sciences historiques et sociales)

Référence électronique

Antoine Laporte, « Holö, Andrej, Lederer, Klaus, Naumann, Matthias, Linke Metropolenpolitik.

Erfahrungen und Perspektiven am Beispiel Berlin », Revue de l'IFHA [En ligne], Date de recension, mis en ligne le 01 janvier 2012, consulté le 22 septembre 2020. URL : http://journals.openedition.org/ifha/ 6534 ; DOI : https://doi.org/10.4000/ifha.6534

Ce document a été généré automatiquement le 22 septembre 2020.

(CIFHA 


\title{
Holö, Andrej, Lederer, Klaus, Naumann, Matthias, Linke Metropolenpolitik. Erfahrungen und Perspektiven am Beispiel Berlin
}

\author{
Antoine Laporte
}

1 L'ouvrage coordonné par A.H., K.L. et M.N. se propose de donner un bilan à la politique municipale menée par le parti Die Linke depuis 2002 et au-delà d'interroger ce que peut recouvrir l'action politique, ici ancrée à gauche, dans une grande ville (la « linke Metropolenpolitik»), et ce, dans un contexte de mondialisation. Il ne s'agit pas ici, comme souvent en géographie urbaine ou en sociologie, d'étudier la ville socialiste ou la ville post-socialiste mais de relire l'évolution générale d'une ville, par le prisme de l'action d'un parti néo-communiste, dans l'opposition dans les années 1990, puis comme participant au gouvernement municipal. Les textes réunis ici sont issus du constat que les espaces urbains sont au centre des attentions de disciplines et d'univers professionnels différents. Au côté des universitaires, sociologues, géographes et philosophes, cet ouvrage donne la parole à des urbanistes et des hommes et femmes politiques locaux.

2 Le livre est divisé en deux parties. La première revient sur le contexte institutionnel, économique et idéologique dans lequel s'inscrit l'action municipale de Die Linke à Berlin. La ville s'est profondément transformée depuis sa position d'espace coupé en deux avant 1989, dont chaque moitié se voulait la vitrine de chacune des deux Allemagnes, à un ensemble urbain unifié très investi par l'État fédéral allemand et traversé par des logiques d'intégration dans les réseaux mondiaux. La deuxième partie évoque plusieurs domaines dans lesquels la politique municipale a une influence plus directe. Le logement tout d'abord, pour lequel un chapitre présente avec beaucoup de précision la montée accélérée des loyers et la privatisation massive des logements sociaux. D'autres chapitres reviennent sur l'action du parti dans la gestion des entreprises publiques (notamment celles en lien avec la distribution de l'eau) ou encore 
sur la position de Die Linke face à l'émergence de la culture comme secteur d'activités incontournable à Berlin.

3 Le point de vue original de l'ouvrage, centré sur l'action de Die Linke, permet de mettre en lumière les conflits entre les échelles de gouvernance. Le parti a fait l'amer constat des limites de l'action locale, cantonnée à quelques domaines comme le logement ou les services publics de proximité. Le livre revient en effet sur la question de la gouvernance des grandes métropoles qui dépasse largement le cas de Berlin. Le parti avoue son impuissance face à des processus urbains qu'il ne peut contrer comme la privatisation des espaces publics, la gentrification et des grands projets pilotés par l'État.

4 Petite ombre au tableau, la portée scientifique de l'ouvrage doit être considérée avec prudence. Si plusieurs chapitres sont des exposés clairs, convaincants et très informatifs, d'autres proposent des analyses plus proches de la prise de position personnelle que de la démonstration. Les auteurs signent ici un livre clairement engagé, mais qui soulève néanmoins des enjeux pertinents sur l'étendue des capacités d'une municipalité dans une grande ville, confrontée à des réalités économiques qui la dépasse.

5 Antoine Laporte (ENS Lyon) 